Écrire

l'histoire

\section{Écrire l'histoire}

Histoire, Littérature, Esthétique

\section{9 | 2019}

\section{L'historien et les langues}

\title{
Écouter les archives
}

Langues vernaculaires et usages sociaux des parlers régionaux au prisme des archives judiciaires d'Ancien Régime

Éva Guillorel

\section{(2) OpenEdition}

1 Journals

Édition électronique

URL : http://journals.openedition.org/elh/1895

DOI : $10.4000 /$ elh. 1895

ISSN : 2492-7457

Éditeur

CNRS Éditions

Édition imprimée

Date de publication : 1 décembre 2019

Pagination : 83-90

ISBN : 978-2-271-12967-3

ISSN : 1967-7499

Référence électronique

Éva Guillorel, «Écouter les archives », Écrire l'histoire [En ligne], 19 | 2019, mis en ligne le 01 décembre 2019, consulté le 16 février 2021. URL : http://journals.openedition.org/elh/1895 ; DOI : https:// doi.org/10.4000/elh.1895 


\section{Écouter les archives}

Langues vernaculaires et usages sociaux
des parlers régionaux au prisme
des archives judiciaires d'Āncien Régime

Le questionnaire envoyé en 1790 par l'abbé Grégoire dans le cadre de la grande enquête préparatoire à son Rapport sur la nécessité et les moyens d'anéantir les patois et d'universaliser l'usage de la langue française, présenté à la Convention nationale en juin 1794, permet de mesurer avec une relative précision l'importance des parlers vernaculaires dans le paysage linguistique de la fin de l'Ancien Régime. Les indications communiquées par les multiples correspondants locaux conduisent ce député fortement impliqué dans le Comité d'instruction publique à dresser le portrait d'un pays largement multilingue, où l'utilisation exclusive du français concerne moins d'un habitant sur dix: une majorité est bilingue, et la plupart des monolingues ne s'expriment que dans leur parler local, en particulier dans les campagnes ${ }^{1}$. Une telle interprétation, peu optimiste quant aux possibilités d'intercompréhension entre les habitants, est certes discutable et influencée par le parcours et le projet de l'abbé Grégoire ${ }^{2}$; mais la multiplicité des langues et des parlers locaux constitue néanmoins une caractéristique indéniable du paysage linguistique du pays. Ce tableau de la fin du xviII ${ }^{\mathrm{e}}$ siècle est lui-même déjà le résultat d'une évolution pluriséculaire en faveur de l'affirmation $d u$ français comme langue officielle de communication.

Les historiens qui s'intéressent à la France d'Ancien Régime doivent donc étudier ses habitants à partir de sources rédigées dans une langue qui est étrangère au plus grand nombre d'entre eux. Pour autant, des références aux parlers locaux apparaissent dans des écrits produits par l'appareil administratif d'État dont ils devraient théoriquement être absents, comme les archives judiciaires. Ce "surgissement du "patois" au cœur même de l'officielle écriture du français ${ }^{3}$ » rappelle d'une part l'omniprésence d'un univers avant tout oral largement occulté dans les sources écrites : les langues et la musicalité de leurs variations dialectales constituent un élément essentiel des "paysages sonores " régionaux - et ce encore bien au-delà de la période révolutionnaire--, dont l'étude entre pleinement dans les renouvellements historiographiques récents sur $l^{\prime}$ histoire des sons et des sens ${ }^{4}$. D'autre 
part, la lecture de certaines procédures criminelles permet de repérer comment l'usage différencié des langues peut être un marqueur des hiérarchies sociales et une source de conflits, tout autant que l'expression privilégiée de tensions à travers des productions littéraires éphémères - pamphlets, vers ou chansons satiriques - dont les archives judiciaires gardent trace.

\section{La place des parlers locaux dans les actes du droit et de l'administration}

L'ordonnance de Villers-Cotterêts établit en 1539 le français, par opposition au latin, comme langue officielle de l'État, entérinant une pratique déjà presque partout acquise. Ce choix est l'aboutissement d'un cheminement de plusieurs siècles autour de la construction d'une politique linguistique qui associe nettement le français à la langue des rois à partir du XIII ${ }^{\mathrm{e}}$ siècle. Il devient la langue des tribunaux, malgré des tensions avec un personnel de justice soucieux de préserver l'usage du latin comme langue savante et spécialisée du droit. Les résistances peuvent également être liées régionalement à l'utilisation de langues vernaculaires distinctes du français dans l'administration au bas Moyen Âge : le picard est largement utilisé dans les chartes municipales entre le $\mathrm{xIII}^{\mathrm{e}}$ et le $\mathrm{xV}^{\mathrm{e}}$ siècle, la chancellerie royale doit transcrire des dépositions reçues en langues d'oc et le parlement de Paris dispose au $\mathrm{xv}^{\mathrm{e}}$ siècle d'une procédure officielle de traduction de pièces en flamand pour traiter les appels en provenance des tribunaux qui émettent des actes en cette langue ${ }^{5}$. Plusieurs affaires rédigées en occitan - lorsque ce parler est le seul compris par les accusés, et donc utilisé pour les interrogatoires - montrent qu'on peut reconnaître à cette langue vernaculaire une valeur juridique: en 1439 , à Figanières, près de Draguignan, le juge accorde à une femme accusée à tort d'avoir usé de magie une mise par écrit du détail de la procédure et des interrogatoires pour prouver sa bonne foi, qui lui est fournie dans sa langue par le greffier du tribunal d'inquisition ${ }^{6}$; en 1497, près de Nîmes, c'est aussi une affaire de sorcellerie qui est presque exclusivement rédigée en langue $\mathrm{d}^{\prime} \mathrm{oc}^{7}$. L'occitan, couramment utilisé dans certains documents écrits comme les actes notariés ou les chartes communales depuis le XII ${ }^{\mathrm{e}}$ siècle, est toujours partiellement en usage dans l'administration au Xvi ${ }^{e}$ siècle : on y a encore recours par endroits pendant plusieurs décennies après l'ordonnance de Villers-Cotterêts pour rédiger des actes d'état civil, des testaments ou des compoix 8 .

Dans les territoires intégrés après le $\mathrm{XVI}^{\mathrm{e}}$ siècle au fur et à mesure de l'agrandissement du royaume, l'utilisation du français pour les documents officiels est affirmée d'emblée et accompagnée d'une réflexion sur la francisation de la population. On assiste toutefois à des périodes de transition, qu'il s'agisse du Béarn, du Roussillon et de la Flandre au cours du XvII ${ }^{e}$ siècle, ou encore de la Lorraine et de la Corse au siècle suivant. 
Le temps d'au moins une génération est nécessaire pour former $\mathrm{du}$ personnel francophone, tandis que les tribunaux ecclésiastiques ou seigneuriaux sont touchés moins brusquement que les juridictions royales, ce qui explique que le flamand reste dominant dans les juridictions inférieures de Flandre jusqu'à la fin de l'Ancien Régime. Seule
l'Alsace bénéficie d'une politique linguistique moins contraignante, qu'il faut comprendre en lien avec la situation religieuse particulière de cet espace fortement marqué par le protestantisme : un arrêt de 1685 y rend obligatoire l'emploi $\mathrm{du}$ français dans l'administration, la justice et le notariat sous peine de nullité et d'amende, mais il n'est guère appliqué 9 .

\section{Procédures judiciaires et références aux langues vernaculaires}

Même lorsqu'elles sont rédigées en français, les procédures criminelles laissent furtivement transparaître la présence des parlers vernaculaires. Le signe le plus évident est la mention d'interprètes. Ceux-ci sont obligatoires depuis la grande ordonnance criminelle de 1670 dès que l'un des prévenus ne connaît pas le français, même si toutes les parties et le juge comprennent la langue locale ${ }^{10}$ : ce cas de figure, fréquent par exemple en Bretagne au début de la période, surtout dans les justices seigneuriales où siègent des magistrats locaux, tend toutefois à s'estomper au $\mathrm{XVIII}^{\mathrm{e}}$ siècle avec la présence croissante de magistrats externes et entièrement francophones ${ }^{11}$. Les mentions de la langue de déposition des témoins, systématiques dans cette région mais pas forcément dans le sud de la France ${ }^{12}$, permettent un traitement sériel pour évaluer le nombre des locuteurs ne maîtrisant pas le français et peuvent être sollicitées pour cartographier des frontières linguistiques dans les espaces de contact entre aires culturelles : cet indicateur n'est toutefois que partiellement fiable, car seuls les monolingues ou ceux qui n'ont qu'une maîtrise trop partielle du français pour témoigner dans cette langue sont ainsi repérés, alors que les comparants bilingues, qui déposent donc en français, ne sont pas identifiables ${ }^{13}$.

Les parlers locaux apparaissent également sous la plume des greffiers lorsque l'interprète est incapable de proposer une traduction ou qu'il semble utile à la compréhension de l'affaire de garder certains termes dans la langue d'origine : toponymes, surnoms, termes techniques ou injures qui perdent leur sens une fois rendus en français sont les premiers concernés. On peut citer parmi de nombreux exemples celui de cette jeune femme de Plougasnou qui en 1724 a tenté d'avorter en buvant « de l'eau de dessus un herbe que l'on appelle en breton lascolen bris que le dit interprette breton nous a déclaré ne pouvoir interpretter en françois", ou de ces jeunes gens près de Carcassonne qui en 1772 vont de maison en maison à l'approche du carnaval "criant de temps en temps et en s'en allant : Couïoul ! Couïoul! » pour se moquer $d^{\prime} u n \operatorname{cocu}^{14}$. Les procédures criminelles du xvIII ${ }^{\mathrm{e}}$ siècle conservées dans 
les archives du parlement de Toulouse contiennent ainsi régulièrement de courtes citations en parler régional, et les témoignages en français comportent de nombreux occitanismes, même parmi les lettrés ${ }^{15}$. Les parlers vernaculaires peuvent aussi être mis par écrit dans des situations où la perte de contrôle entraîne le retour à la langue maternelle : dans le procès de Louis Gaufridy, prêtre de Marseille accusé de sorcellerie en 1611 , les nombreux imprimés qui médiatisent largement l'affaire ne mentionnent pas d'indications linguistiques; mais les procès-verbaux manuscrits du procès précisent que, lors d'une séance d'exorcisme, une des filles possédées dit que Dieu l'a «contraint de dire toutes ces choses en langue provençale, pour les ignorants ", tandis que le prêtre oublie son latin et se met à parler sa langue maternelle sous l'effet de la torture ${ }^{16}$.

Par ailleurs, des mentions de lecture à voix haute de proclamations, règlements ou sentences énoncés à la fois en français et en langue régionale montrent que l'efficacité - donc la compréhension du message par toute la population - est la première des préoccupations. Elles rappellent aussi l'importance de l'oralité au cours de procédures judiciaires marquées par toute une gamme de manifestations sonores, tant de la part des juges que des parties en présence et du public (du murmure à la clameur en passant par le dialogue, l'injure, la rumeur, la proclamation ou le cri public), qui forment un " paysage sonore partagé » entre justice et justiciables ${ }^{17}$.

Dans le domaine de la justice civile, les références aux parlers locaux sont encore plus présentes: l'analyse de registres de factums de la deuxième moitié du XvIII $^{\mathrm{e}}$ siècle dans le sud de la France montre que les occitanismes ne sont pas utilisés par maladresse, ou par ignorance $\mathrm{du}$ terme français correspondant, mais de façon délibérée lorsqu'ils permettent un discours plus précis ou plus efficace, voire plus éloquent: unités de mesure locales, termes en lien avec le commerce, la terre ou les métiers, sont les usages les plus fréquents, à l'image de ce mémoire sur un procès au sujet de la fermeture d'une tannerie à Grasse en 1754 qui est truffé de termes en langue d'oc ${ }^{18}$.

\section{Hiérarchies et conflits autour de l'usage des langues vernaculaires}

En dehors de la langue des procédures, les mentions de l'utilisation de parlers locaux dans les procès surgissent lorsque l'usage différencié des langues est révélateur de tensions. Bien plus finement que les indications des greffiers sur la langue des déposants, ces éléments permettent de cerner des profils linguistiques aux contours mouvants qui traduisent des hiérarchies sociales, mais aussi des proximités culturelles au sein des communautés. À Maël-Pestivien en 1735 , une affaire de fratricide sur fond de conflit successoral met ainsi aux prises une famille de petite noblesse qui adapte le choix de sa langue selon le public visé : en breton pour menacer leur mère, mais en français devant les domestiques pour 
s'assurer qu'ils ne comprennent pas ce qu'ils disent; ils sont loin de se douter que la filandière qui travaille pour eux au manoir, bien que déposant en breton et ne sachant pas signer, comprend suffisamment de français pour se rendre compte du meurtre qui se prépare et témoigner à charge contre les assassins. Une telle affaire met donc en scène à la fois une noblesse locale plurilingue pour qui le breton peut être une langue de culture autant que le français et le latin, et des domestiques qui intègrent au contact de ces milieux nobiliaires, au moins de façon partielle et passive, une langue française qu'ils ne pratiquent pas par ailleurs ${ }^{19}$.

D'autres procédures montrent comment l'usage différencié des langues peut être source de conflits, soit par manque de maîtrise - comme les cas de prêtres se mettant à dos la communauté paroissiale parce qu'ils ne parlent pas correctement le parler local -, soit par usage inapproprié d'une langue eu égard aux attentes sociales - comme le recours à des propos ou injures en langue vernaculaire pour minimiser l'écart social entre paysannerie et noblesse ${ }^{20}$. À Pernes en Artois en 1539, le simple fait de trinquer en flamand et les remarques désobligeantes que suscite l'usage de cette langue par des témoins suffisent à conduire à la violence meurtrière $^{21}$. Les plaignants insistent souvent sur la plus grande force et violence que revêt un discours s'il est prononcé dans la langue locale, surtout dans le cas des injures et propos diffamatoires: un notable de Pédernec, près de Guingamp, explique ainsi dans sa plainte en 1773, suite aux propos calomnieux qui l'ont visé, que "ces injures en françois sont certainement des plus graves et portent à la réputation du suppliant une atteinte trop irréparable pour ne pas être poursuivie extraordinairement, en breton elles ont encore plus d'énergie ${ }^{22} »$.

Il n'est pas étonnant que ce dernier exemple s'inscrive dans le cadre d'une affaire liée à une chanson diffamatoire. Ces affaires ont l'intérêt de souvent conserver comme pièces justificatives des copies des écrits mis en cause. Yves Castan précise même que la chanson constitue le seul type de textes qui persistent en langue d'oc dans les procédures du $\mathrm{xvIII}^{\mathrm{e}}$ siècle $^{23}$. Les exemples sont multiples: à Pamiers en 1735, des commis de boutique et des garçons savetiers sont reconnus comme les auteurs de deux chansons en occitan, dont l'une est écrite et imprimée; à Castelnaudary la même année, une quinzaine de femmes accusent une habitante de prostituer sa fille, dans des chansons en langue locale dont certains couplets sont recopiés dans la procédure ${ }^{24}$. Les charivaris, bien présents dans les procès criminels de Guyenne, Gascogne et Languedoc, mettent également en scène la composition et la performance orale de chansons en parler régional, parfois mises par écrit, distribuées et placardées, ou encore interprétées à la fois en français et en gascon pour assurer une diffusion dans tous les milieux sociaux ${ }^{25}$. En 1697 au Trévoux, dans le sud-ouest de la Bretagne, une affaire implique le curé de la paroisse, qui envoie deux jeunes gens chansonner un débitant de vin avec des vers satiriques imitant un cantique : ils sont affichés sur un chêne au vu de tous et peuvent ainsi être ajoutés à la procédure ${ }^{26}$. Dans le Lyonnais, c'est cette fois une chanson "en patois bressan" qui est à l'origine d'une rixe, à la fin des années 1780, parce qu'elle est jugée injurieuse par l'un des convives qui «se 
scandalisa de cette chanson et prétendit qu'on cherchait par là à le mortifier parce

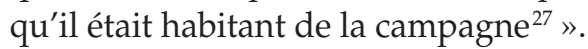

Les chansons en langue vernaculaire peuvent donc exacerber des hiérarchies et des tensions sociales tout en redonnant aux parlers locaux leur place essentielle, mais autrement peu visible dans les archives judiciaires et administratives. Ces productions culturelles sont doublement subversives puisqu'elles remettent publiquement en cause la réputation d'individus - en visant le plus souvent des notables locaux - tout en transgressant les normes officielles de l'écrit par le recours à une langue autre que le français. Elles ont d'autant plus d'intérêt que les procédures criminelles détaillent souvent avec précision les auteurs, les lieux et les circonstances de la performance orale, de même que les réactions de l'auditoire et des autorités. L'affaire de Pédernec en 1773 en est un bon exemple ; la plainte déposée par Guillaume Scolan détaille toute la stratégie de communication des auteurs du chant qui le ridiculisent en montrant l'efficacité et par conséquent l'ampleur du préjudice subi : le choix de la langue, bien sûr, mais aussi de la mélodie - sur un air déjà connu - ou des lieux de performance - le dimanche à la sortie de l'église pour attirer une large foule -, tout concourt à renforcer $l^{\prime}$ offense subie ${ }^{28}$.
"Pourquoi un historien de la culture devrait-il écrire sur le langage? Et pourquoi ne pas laisser ce sujet à des linguistes? » En posant ces questions comme point de départ de son essai Languages and Communities in Early Modern Europe, Peter Burke défend une « histoire culturelle du langage » attentive à ce que les usages des langues, les circulations et emprunts linguistiques ou encore les variations entre registres langagiers révèlent sur les sociétés et les changements culturels qui les animent dans l'Europe moderne ${ }^{29}$. À une échelle plus modeste, l'analyse des mentions de parlers vernaculaires dans les archives judiciaires françaises, tant dans la langue des procédures que dans les références à l'usage de ces langues dans les plaintes, dépositions ou interrogatoires de témoins, permet de mettre au jour les enjeux socioculturels qui tournent autour de l'usage différencié d'une multiplicité de parlers dont l'interaction fluide se nourrit de rapports de force, de hiérarchies et de tensions. Ainsi peut-on dresser un tableau subtil de situations d'échange, de conflits ou de diglossie linguistique dans une France d'Ancien Régime profondément marquée par un multilinguisme pourtant peu apparent dans les sources qui nourrissent la réflexion des historiens.

\section{Notes}

1 Michel de Certeau, Dominique Julia, Jacques Revel, Une politique de la langue. La Révolution française et les patois: l'enquête de Grégoire, Gallimard, 1975; Augustin Gazier (édit.), 
Lettres à Grégoire sur les patois de la France (1790-1794), Paris, A. Durand et PedoneLauriel, 1880.

2 David A. Bell, "Tearing Down the Tower of Babel. Grégoire and French Multilingualism ", dans Jeremy D. PopKIN, Richard H. POPKIN (dir.), The Abbé Grégoire and His World, Dordrecht, Kluwer, 2000, p. 109-128.

3 Philippe Martel, "L'occitan », dans Georg Kremnitz (dir.), Histoire sociale des langues de France, PUR, 2013, p. 513.

4 Parmi de nombreux travaux récents sur ce sujet, voir Arlette FARGE, Essai pour une histoire des voix au XVIII siècle, Bayard, 2009; Laurent Hablot, Laurent Vissière (dir.), Les Paysages sonores du Moyen Âge à la Renaissance, PUR, 2015, DOI : 10.4000/books.pur.47096 ; Constance Classen (dir.), A Cultural History of the Senses, Londres, Bloomsbury, 2014, 6 vol.

5 Serge Lusignan, La Langue des rois au Moyen Âge. Le français en France et en Angleterre, PUF, 2004 ; ID., "Histoire et sociolinguistique: le français picard et les administrations publiques du nord-ouest de l'Europe », dans Jean-Marie Bertrand et al. (dir.), Langue et Histoire, Publications de la Sorbonne, 2011, p. 91-101.

6 Roger AubenAs, La Sorcière et l'Inquisiteur. Épisode de l'Inquisition en Provence, 1439, La Pensée universitaire, 1956.

7 Pedro Casado, Un procès de sorcellerie dans la Vaunage (Calvisson, Clarensac) à la fin $d u$ XV siècle, C. Lacour, 2005.

8 Philippe Martel, «L'occitan, le latin et le français du Moyen Âge au xvI ${ }^{\mathrm{e}}$ siècle ", dans Henri Boyer, Philippe Gardy (dir.), Dix Siècles d'usages et d'images de l'occitan. Des troubadours à l'Internet, L'Harmattan, 2001, p. 65-117.

9 Herman VAN Goethem, «La politique des langues en France, 1620-1804 », Revue du Nord, t. $71, n^{\circ} 281,1989$, p. $437-460$.

10 Christiane Plessix-Buisset, Le Criminel devant ses juges en Bretagne aux $\mathrm{XVI}^{e}$ et XVII siècles, Maloine, 1988, p. 312.

11 Philippe JARnoux, «Le personnel des justices seigneuriales en basse Bretagne au XVIII $^{\mathrm{e}}$ siècle ", dans François BRIZAY, Antoine Follain, Véronique SARrazin (dir.), Les Justices de village. Administration et justice locales de la fin du Moyen Âge à la Révolution, PUR, 2003, p. 297-310, DOI : $10.4000 /$ books.pur.19028, ici p. 307-308.

12 Yves Castan, «Les Languedociens du XVIII ${ }^{\mathrm{e}}$ siècle et l'obstacle de la langue écrite ", dans Actes du $96^{e}$ congrès national des Sociétés savantes (Toulouse, 1971). Section d'histoire moderne et contemporaine, t. 1, France du Nord et France du Midi, Bibliothèque nationale, 1976, p. 73-84.

13 Fañch Roudaut, «Les archives judiciaires au service de la géographie linguistique: l'exemple de la basse Bretagne au XviII ${ }^{\mathrm{e}}$ siècle ", Bulletin de la Société archéologique du Finistère, t. 109, 1981, p. 209-227.

14 Éva Guillorel, «Langues, hiérarchies et conflits dans la Bretagne d'Ancien Régime: l'apport des archives judiciaires », dans Gildas Buron, Herve Bihan, Bernard Merdrignac (dir.), À travers les îles celtiques. Mélanges à la mémoire de Gwénaël Le Duc, PUR, 2008, p. 354-356 ; Daniel FABRE, «Familles. Le privé contre la coutume", dans Philippe ArIÈs, Georges Duby (dir.), Histoire de la vie privée, t. 3. De la Renaissance aux Lumières, Seuil, 1986, p. 564-566.

15 Yves Castan, Honnêteté et relations sociales en Languedoc (1715-1780), Plon, 1974, p. 115-116.

16 Jean Eygun, Au risque de Babel. Le texte religieux occitan de 1600 à 1850, Association d'étude du texte occitan, 2002, p. 268-271.

17 Romain Telliez, « À cor et à cri. Le paysage sonore de la justice, en France à la fin du Moyen Âge ", dans Laurent HABLot, Laurent Vissière (dir.), op. cit., p. 73-98, DOI : 10.4000/books. pur.47108. Telliez se réfère à une expression proposée par Arlette FARGE dans son Essai pour une histoire des voix..., op. cit., p. 260.

18 Georges Gibelin, « Les occitanismes dans les textes des plaideurs de la seconde moitié du $\mathrm{xVIII}^{\mathrm{e}}$ siècle ", Lengas. Revue de sociolinguistique, $\mathrm{n}^{\circ} 17,1985$, p. 37-47.

19 Archives départementales des Côtes-d'Armor, juridiction seigneuriale de Callac, B 228.

20 Éva Guillorel, «Du bon usage des langues dans la Bretagne d'Ancien Régime ", dans François BRIzAY (dir.), Les Formes de l'échange. 
Communiquer, diffuser, informer de l'Antiquité au XVIII ${ }^{e}$ siècle, PUR, 2012, p. 61-64.

21 Robert Muchembled, La Violence au village. Sociabilité et comportements populaires en Artois du XVe au XVII siècle, Turnhout, Brepols, 1989, p. 128.

22 Éva Guillorel, La Complainte et la Plainte. Chanson, justice, cultures en Bretagne (XVIXVIII siècles), PUR, 2010, p. 221-223.

23 Yves Castan, op. cit., p. 118.

24 Ibid., p. 156 ; Daniel FABRE, art. cit., p. 556.

25 Nicole Castan, "Contentieux social et utilisation variable du charivari à la fin de l'Ancien Régime en Languedoc », dans Jacques LE
Goff, Jean-Claude Schmiтt (dir.), Le Charivari, EHESS/Mouton, 1981, p. 198-202.

26Gwennole LE MENN, «Une chanson satirique du xvII $^{\mathrm{e}}$ siècle en dialecte de HauteCornouaille ", Annales de Bretagne, vol. 75, no 4, 1968, p. 693-704, DOI : $10.3406 /$ abpo.1968.2494, ici p. 693-696.

27 Archives départementales du Rhône, juridiction seigneuriale d'Anse, 2 B 61.

28 Éva GuILlOREL, op. cit., p. 221-223.

29 Peter BuRKe, Languages and Communities in Early Modern Europe, Cambridge University Press, 2004, p. 1 (notre traduction); voir aussi Peter Burke, Roy Porter (dir.), The Social History of Language, Cambridge University Press, 1987. 